\title{
Lengua y literatura en las aulas de español como lengua extranjera en el sistema educativo de Camerún
}

\author{
André-Marie Manga \\ Université de Yaundé I (E. N. S.) \\ madrema@rocketmail.com
}

RESUM

Llengua i literatura a les aules d'espanyol com a llengua estrangera en el sistema educatiu de Camerun

Aquest article va dirigit essencialment als docents, futurs professors i alumnes que tenen una preocupació constant per assolir una bona formació, un bon reciclatge. L'autor voldria posar en relleu el caràcter indissociable de la llengua i la literatura, com a pilars fonamentals tant per a la comunicació i expressió, com per a la construcció de la reflexió. De fet, es considera que si els docents no impartissin separadament les nocions de llengua i literatura, aconseguirien que els seus alumnes dominessin molt millor la competència comunicativa. Per tant, se suggereix que la planificació i la tasca educativa dels discents es concretin tenint en compte aquests dos elements de la construcció dels coneixements, per garantir una millor formació a l'ésser humà.

Paraules Clau

Llengua, literatura, docència.

\section{RESUMEN}

Lengua y literatura en las aulas de español como lengua extranjera en el sistema educativo de Camerún

Este artículo va dirigido esencialmente a los docentes, futuros profesores y alumnos cuyo deseo sigue siendo una buena formación, un buen reciclaje. El autor quisiera poner de relieve el carácter indisociable de la lengua y la literatura, como pilares fundamentales tanto para la comunicación y expresión, como para la construcción de la reflexión. De hecho, se piensa que si los docentes no impartiesen separadamente las nociones de lengua y literatura, conseguirían que sus alumnos tuvieran un dominio mucho mejor de la competencia comunicativa. Por consiguiente, se sugiere que la planificación y la labor educativa de los discentes se concreten teniendo en cuenta estos dos elementos de la construcción de los conocimientos, para garantizar una mejor formación al ser humano.

Palabras Clave

Lengua, literatura, docencia.

RÉSUMÉ

Enseignement de la langue et la littérature en espagnol langue étrangère dans le système éducatif camerounais

Cet article se propose surtout d'attirer l'attention des enseignants, des professeurs en formation et des élèves, dont le souci permanent reste la bonne formation et le recyclage. L'auteur y souligne le 
caractère indissociable de la langue et la littérature, qui sont la base de la communication et de la construction de la pensée. Il pense humblement que si les enseignants ne dispensaient pas séparément les cours de langue et littérature, leurs élèves maîtriseraient nettement mieux la compétence de communication. Aussi suggère-t-il que la planification et l'action éducative se réalisent toujours avec ces deux composantes de la construction des connaissances, pour assurer une meilleure formation à l'être humain.

\section{Mots-CLÉS}

Langue, littérature, enseignement.

\section{Abstract}

\section{Teaching language and literature in Spanish as foreign language classrooms in Cameroon}

This article essentially concerns teachers, student teachers and students who are supposed to be well-trained and/or recycled. The author would like to put emphasis on the inextricable relationship between language and literature, which are the basis of communication and knowledge construction. He states that students would acquire better communicative competences if teachers avoided teaching language and literature separately. Thus, it is suggested that the lesson planning and the educative action should always be accomplished taking into consideration these two entities of knowledge construction in order to ensure a better training for the human being.

\section{KEYWORDS}

Language, literature and teaching.

\section{Introducción}

La lógica de la reflexión intelectual suele exigir aclaraciones de conceptos antes de su uso en los discursos orales o escritos, así como en las meras interacciones. Por eso pensamos que es indispensable definir de nuevo el término educación en estas líneas, antes de llegar al debate sobre la enseñanza de la lengua y la literatura. La educación se define generalmente como la orientación del desarrollo físico, moral e intelectual de un niño o de un adolescente en un sentido estimado mejor. Es también la transmisión de valores, virtudes y aptitudes de una generación de individuos a otra. Moumouni (1998:24-25) hace un recorrido de los distintos rasgos de la educación tradicional en África, y diseña la educación como el desarrollo de las aptitudes físicas de un niño o adolescente y, sobre todo, pone de realce el importante papel de estas aptitudes durante los primeros años de la existencia del ser humano. A continuación, se refiere a la formación de su carácter, a la adquisición de las cualidades morales como objetivos primordiales en la educación tradicional africana. Aparentemente, no se considera bastante la formación intelectual en este modelo, pero cabe señalar la adquisición de los conocimientos generales, la cultura general en historia, geografía, filosofía, etc. Se notan estrechas relaciones entre esta educación tradicional y algunas realidades de la escuela moderna. En el entorno en que se desarrolla esta reflexión, el sistema educativo funciona con el apoyo de la Loi d' Orientation de l'Education (1998). En uno de sus artículos, se pone de relieve la necesidad de formar a ciudadanos totalmente integrados en su propia cultura, y al mismo 
tiempo, abiertos espiritualmente a las influencias exteriores. El programa ${ }^{1}$ de enseńanza/aprendizaje del español como lengua extranjera en la Enseñanza Secundaria (2000) privilegia en sus objetivos el estudio del español como lengua; es decir lengua contemporánea, lengua funcional, en su forma oral, para favorecer y agilizar la expresión verbal, la interacción, la comunicación. Además, se estudia el español en la literatura para la formación moral e intelectual del alumno para que pueda adquirir los conocimientos necesarios, y desarrolle la inteligencia, el sentido estético, crítico y la formación de una conciencia moral. A partir de los diseńos curriculares, se piensa en las demandas sociales que recaen en algunas materias arriba mencionadas. En unas palabras, se intenta adaptar la cultura educacional a las necesidades de cada entorno. De hecho, se piensa que si los docentes no impartiesen separadamente las nociones de lengua y literatura, conseguirían mejor su objetivo en el dominio de la competencia comunicativa de los alumnos.

\title{
Programas de formación en lengua, literatura y civilización
}

Antes de entrar en el análisis propiamente dicho de la docencia del español en Camerún, pensamos que vale la pena echar un vistazo en lo que se propone en los programas de enseńanza/aprendizaje del francés, lengua oficial y de escolarización en nuestro entorno. El documento Programmes. Langue et Littérature 2nd Cycle (1994) de la Enseñanza Secundaria (General y Técnica) en Camerún, presenta los objetivos específicos detallados, para las clases de francés, entre los que se destacan los deseos de:

\begin{abstract}
S'exprimer aisément et correctement, oralement et par écrit; manier des structures grammaticales complexes et un vocabulaire riche pour traduire sa pensée, ses sentiments ou des concepts; reconnaitre et caractériser divers niveaux de langue et en faire un usage approprié ; commenter, discuter, résumer un document écrit, oral ou iconique; prendre part à un débat; se constituer une documentation (prendre des notes, les classer, les utiliser), définir une situation de communication; expliquer comment s'élabore un message en relation avec une situation donnée et un objectif à atteindre; reconnaitre et manier les articulations logiques, explicites et implicites d'un texte; manier les techniques de l'argumentation; repérer un type de texte, définir son statut, analyser sa spécificité; poser et exploiter concrètement les principales questions qui peuvent guider une lecture méthodique et aider à construire un commentaire de texte.
\end{abstract}

Nos importa considerar otra referencia cuyo contenido nos parece en total simbiosis con lo que se espera que domine el alumno, tras haber estudiado durante cinco años en la Enseñanza Secundaria la lengua y la cultura españolas. Se trata del conjunto de los objetivos generales del Diseño Curricular Base (DCB), planteados por Nusbaum y Bernaus (2001:126-127), al finalizar

1. Documento oficial firmado en el año 2000 por el ministro de la Educación Nacional de Camerún, el Profesor Joseph Owona. Dicho documento desempeña el mismo papel que el currículum como marco general de planificación, actuación y evaluación en el ámbito de la enseńanza y aprendizaje; su objetivo principal es facilitar laintegración y coherencia de las decisiones que se adoptan y de las actividades que se llevan a cabo. Comprende la fijación de unos fines y objetivos, la selección de unos contenidos, la opción por una metodología y el establecimiento de unos criterios de evaluación; estos cuatro componentes del currículo derivan de una determinada comprensión de la naturaleza de la lengua, de su aprendizaje y de los principios básicos educativos y culturales predominantes. La coherencia es el principio fundamental del currículo, puesto que existe una dependencia mutua entre los distintos factores que intervienen en la situación de enseñanza y aprendizaje, según lo define el Diccionario de términos clave en ELE. 
en España la Educación Secundaria Obligatoria. En el área de Lenguas Extranjeras, los alumnos habrán desarrollado la capacidad de:

1. Comprender la información global y especifica de mensajes orales en la lengua extranjera relativos a las situaciones de comunicación más habituales en la vida cotidiana, emitidos directamente por hablantes de la misma o por medios de comunicación.

2. Producir mensajes orales en la lengua extranjera utilizando recursos lingüísticos (...) en las situaciones de comunicación más habituales en la vida cotidiana para conseguir que la comunicación sea fluida y satisfactoria, mostrando una actitud de respeto e interés por comprender y hacerse comprender.

3. Leer de forma comprensiva y autónoma, obteniendo informaciones globales y específicas, textos escritos de un nivel adecuado a las capacidades e intereses de los alumnos, relacionados con situaciones de comunicación escolares y extraescolares.

4. Utilizar la lectura de textos escritos con finalidades diversas valorando su importancia como fuente de información, disfrute y ocio y como medio de acceso a culturas y formas de vida distintas de las propias.

5. Producir textos escritos de utilidad en la vida cotidiana relacionados con las necesidades e intereses propios, con la corrección suficiente para asegurar su comprensión por los destinatarios.

6. Expresar oralmente y por escrito en la lengua extranjera mostrando interés por comprender el funcionamiento del sistema lingüístico como medio para mejorar las producciones propias.

7. Situar la lengua extranjera estudiada con referencia a su utilización actual en las relaciones internacionales y en otros ámbitos de la actividad humana (científico, tecnológico, literario, laboral, etc.), valorando positivamente la ayuda que supone el conocimiento de la misma para participar en ellos.

Aquí, se anotan unas opiniones en adecuación con las metas que fijan los expertos para la enseñanza/aprendizaje de las lenguas-culturas extranjeras en el Plan Curricular del Instituto Cervantes (1993:5). De hecho,

El plan curricular del Instituto Cervantes concibe la enseñanza como una actividad que no se limita a la mera transmisión de conocimientos, sino que se orienta hacia el desarrollo de nuevas aptitudes y capacidades en los alumnos. Para lograr este desarrollo, es necesario contar con la participación activa de los alumnos en múltiples actividades de aprendizaje. Por otra parte, en lo que respecta al establecimiento de los objetivos, el currículo exige un análisis de las necesidades de los alumnos en los diversos ámbitos que afectan al proceso de aprendizaje. Como consecuencia de todo lo anterior, el Plan Curricular parte del respeto a las características individuales de cada persona y reconoce la necesidad de que los alumnos tengan una función activa en clase y se conviertan en responsables de su propio proceso de aprendizaje.

Existe una verdadera dificultad en la enseñanza/aprendizaje de las lenguas extranjeras, si se deja que la teoría se oponga a la práctica en las actividades de clase. Se trata de una discrepancia que puede solucionarse con la insistencia sobre ambos aspectos, a partir de la formación del profesorado. Aprender una lengua supone referirse siempre a las cuatro destrezas tradicionales que son: la comprensión oral, la expresión oral, la comprensión lectora y la expresión escrita. La solución a la adquisición de las cuatro destrezas aparece en el dominio de las funciones del profesor que especifica Cortés Moreno (2000:59) acerca del profesor, cuando afirma que el profesor tiene el secreto de la enseńanza y el alumno tiene el secreto del aprendizaje: ambos son importantes por igual, cada uno 
en sus funciones. En esta reflexión, no nos interesan las funciones del alumno en el acto didáctico. Intentaremos enumerar los distintos roles que desempeña el maestro en la realización concreta de las actividades de clase. ¿Pues, cuáles son las funciones del profesor? Además de la función tradicional de fuente de conocimientos - es efectivamente la primera fuente de conocimientos de sus alumnos- y puede asumir, para que tenga lugar con contundencia la enseñanza/aprendizaje de las lenguas-culturas extranjeras, dentro del aula, múltiples papeles tales como: director, organizador, controlador, evaluador, consejero, asesor, animador, modelo lingüístico y cultural, presentador de muestras lingüísticas y culturales, diseñador de materiales, observador, investigador en el aula, participante en las tareas (como un alumno más) aprendiente (un aprendiz de lenguas puede ser especialista en su propia lengua o en cualquier otro ámbito: música, cocina...)

Esta reflexión, cuya base es la práctica pedagógica, tiene como meta llamar la atención de los actores del proceso de enseñanza/aprendizaje de las lenguas, para que la lengua y la literatura sean las herramientas básicas del desarrollo intelectual y de la formación académica. De esta manera, se puede suponer que si los agentes del sistema educativo tuvieran en cuenta el verdadero papel de la lengua y la literatura dentro del aula, se formaría mejor a los discentes. Parece que se trata de la misma preocupación la que anima a Lázaro Carreter et al. (1996), cuando hablan de fondo y forma en una explicación de texto, con dos objetivos específicos: fijar con precisión lo que dice el texto, y dar razón de cómo lo dice. Pues separar la forma y el fondo para su estudio sería tan absurdo como deshacer un tapiz para comprender su trama: obtendríamos como resultado un montón informe de hilos. De hecho, en el aprendizaje formal y, sobre todo, en los niveles básicos, la lengua y la literatura aparecen como dos ejes fundamentales a partir de los cuales se construye todo el éxito posterior que consiguen los alumnos. La didáctica de la lengua extranjera obedece al mismo funcionamiento. Ahí, los investigadores y los profesionales de la enseñanza suelen poner de relieve la enseñanza-aprendizaje de la lengua y la literatura para desarrollar en los alumnos las habilidades, las destrezas comunicativas, y los saberes y actuaciones culturales, en una palabra, la competencia comunicativa $^{2}$ que necesitan en la lengua meta, y que les facilitan la expresión oral en situaciones reales de comunicación. A este respecto, Lomas (1999: 133) reconoce:

Cuando un profesor o una profesora entran en un aula lo hacen con la intención de que los alumnos y las alumnas aprendan (o aprendan a hacer) determinadas cosas. En el caso del profesorado de Lengua y Literatura, es obvio que de lo que se trata (...) es que los alumnos y las alumnas adquieran un conjunto de destrezas comunicativas (hablar, escuchar, leer, entender, escribir) que les permitan comportarse comunicativamente de una manera adecuada, eficaz y competente en las diversas situaciones comunicativas de la vida cotidiana. De ahí que el objetivo esencial de la educación lingüística y literaria (...) sea el desarrollo dela competencia comunicativa(expresiva y comprensiva) de los alumnos y de las alumnas.

Se trata de un objetivo claramente expuesto, pero su realización exige la puesta en marcha de distintos parámetros didácticos, la voluntad de los alumnos, y otros factores de índole socioeconómica y política. Además, se sabe que la lengua hablada en un contexto dado no es homogénea,

2. La noción de competencia comunicativa se define en torno a cuatro parámetros según Hymes: 1) La posibilidad que se refiere a la gramaticalidad de los enunciados. 2) La verosimilitud, o la probabilidad de que un enunciado ocurra. 3) La propiedad: Hymes defiende que es posible articular un enunciado perfectamente gramatical, que además es producido con normalidad por los hablantes nativos de una lengua. 4) Que algo suceda en la actuación; es decir que el enunciado se produzca en la comunicación real. 
ya que los distintos registros se corresponden con los respectivos grupos sociales, y los entornos de interacciones de los hablantes. Por una parte, esta diversidad de registros existentes puede conducir al docente a equivocarse en el momento de elegir la lengua que debe enseñar en su clase, y por otra, los alumnos pueden empezar a tener confusiones y, a veces, utilizar cualquier registro en sus producciones orales y escritas. Una vez más, se llama la atención de los formadores, para que estén siempre en alerta, y sepan distinguir los registros lingüísticos que utilizan sus alumnos. Para evitar estas dificultades, Cortés Moreno (2000: 28) apunta: "A modo de sugerencia, entendemos que el prioritario es el registro formal, pero ello siempre no será así en todas las situaciones de aprendizaje”. A continuación, el mismo investigador propone y aclara el registro contemporáneo como se lee en el esquema siguiente:

Cuadro i: Registro lingüístico

\begin{tabular}{|l|l|l|l|l|}
\hline \multicolumn{2}{|l|}{ Registro contemporáneo } & \multirow{2}{*}{$\begin{array}{l}\text { Registro } \\
\text { clásico }\end{array}$} \\
\cline { 1 - 3 } $\begin{array}{l}\text { Registro } \\
\text { popular }\end{array}$ & \multicolumn{2}{|c|}{ Registro estándar } & $\begin{array}{l}\text { Registro } \\
\text { literario }\end{array}$ & \\
\cline { 2 - 3 } & Registro familiar & Registro formal & Registro culto & \\
\hline
\end{tabular}

Lengua y literatura constituyen los dos pilares esenciales de los contenidos que se diseñan y se comentan dentro de las aulas de español como lengua extranjera. El Plan Curricular del Instituto Cervantes (1993: 81) propone como contenido temático: lengua, cultura y sociedad; y ya integra varios aspectos de la vida cotidiana para favorecer la comunicación real en las interacciones de los alumnos. Los temas de los textos literarios constituyen en esta sección el marco situacional en el que aparecen las diferentes muestras de lengua (textos impresos, diálogos, materiales auténticos, etc.), su presencia en el currículo es, pues, implícita, aunque en algunos casos puede ser necesario un tratamiento explicito.

La literatura propiamente dicha, constituye una de las más serias dificultades que experimentan los docentes de español lengua extranjera en Camerún, quizá porque no se tiene claro cuál es su papel, ni sus objetivos en el proceso educativo. En la Enseñanza Secundaria precisamente, la poesía que forma parte de la literatura sigue siendo una de las asignaturas más herméticas para los alumnos y los docentes. No es éste el lugar adecuado para llevar a cabo una reflexión sobre la poesía. Procuraremos hablar de la literatura en general, con la intención de recordar su importante papel a todos los que se dedican a la enseñanza/aprendizaje de las lenguas-culturas extranjeras. Los estudiosos coinciden en que todos los seres humanos tienen el deseo de expresarse, de comunicar. Romera Castillo (1992: 145) justifica en los términos siguientes el papel de la literatura en la educación:

(...) la literatura es un instrumento válido en la formación de los individuos porque se proyecta sobre la problemática vital de los individuos, sirve para transformar la realidad y, a la vez, es instrumento de goce y placer. Con la literatura se pueden conseguir unos objetivos: a) De conocimiento: proporcionando una serie de datos, informaciones que pueden contribuir al mejor conocimiento tanto de la historia cultural de una región, un país, una nacionalidad o un universo, como al hecho histórico al que éstos se refieren. b) De desarrollo: El principal objetivo de toda enseñanza es formar al alumno para que sea un hombre completo (desarrollo de su inteligencia y personalidad), y la literatura se puede utilizar en su proyecto vital. 
De ahí, se deduce que la literatura es una necesidad para todo el mundo. En relación con esta observación, se desvela que su contenido debe diseñarse para alcanzar fines sociales, y necesidades inmediatas de los alumnos. La enseñanza de la lengua se realiza también con la idea de la inserción profesional de los alumnos en la sociedad. El docente lo tiene que saber y evitar así cualquier desviación de su tarea. Desde la perspectiva de las actuales normas de enseñanza de la lengua extranjera, el objetivo fundamental y prioritario no es formar a filólogos o a lingüistas expertos. Lo que debe hacer el docente es enseñar a sus alumnos, como sugiere González Nieto (2001:19), no para que se conviertan en filólogos o en lingüistas, sino, para que adquieran de forma progresiva las capacidades verbales que la sociedad demanda a las personas adultas, es decir, que se conviertan en usuarios competentes del idioma. Se consigue más fácilmente este proceso estudiando la lengua a partir de textos literarios. También se invoca la necesidad de que se conozca el patrimonio literario y socio-cultural. Teniendo en cuenta estos dos aspectos, algunos docentes, porque no coinciden en la manera cómo llevar a cabo su tarea, empiezan a enseñar Lengua y Literatura por separado. En la actualidad, hay un cambio cuyas implicaciones son vigentes en los procesos de enseńanzaaprendizaje de la lengua. En ambos casos, siendo el mismo objetivo: favorecer la comunicación oral, la enseñanza de la lengua y de la literatura debe centrarse en la formación lingüística y sociocultural, y poner fin a la dicotomía entre ambos conceptos; si bien existen algunos irreductibles. En efecto, con el desarrollo del análisis del discurso y de la perspectiva pragmática, muchos profesores siguen preguntándose si no hace falta volver a la tradicional forma de enseñanza que recomendaba la separación entre lengua y literatura. En otros términos, se trata de precisar si los usos lingüísticos son polos literarios. A partir de esta observación y, teniendo en cuenta la competencia comunicativa como objetivo prioritario de la enseńanza/aprendizaje de las lenguas, ya no queda duda sobre la necesidad de considerar simultáneamente la lengua y la literatura en la formación e inserción social de los alumnos, como se nota en las siguientes palabras de Mendoza Fillola et al. (1996: 220):

La correlación entre competencia lingüística y competencia literaria nos permite apreciar la continuidad de ambas y las implicaciones de enseñanza/aprendizaje que ello conlleva. La competencia lingüística es observable en el resultado de la actuación del hablante, en su saber - hacer productor (en cuanto interacción, expresión, creación o comprensión). En cambio, para detectar la competencia literaria, por lo que se refiere al ámbito escolar, atenderemos a la capacidad de comprensión-interpretación de los textos. (...) Vemos, pues que la competencia comunicativa ha resultado ser un concepto clave para organizar muy diversos objetivos y contenidos propios de la DLL.

Además, si queremos conseguir la adquisición de habilidades expresivas, el análisis del diálogo y de la conversación pasa a ser la piedra angular de la actividad docente, para que el profesor prepare y adapte los textos, y que los alumnos descubran las interacciones en situaciones concretas de la vida real. A partir de esta metodología, se vuelve al objetivo prioritario que quieren alcanzar los que se dedican al aprendizaje de otras lenguas: poder interactuar en dichas lenguas. Por eso abundan diálogos en manuales de enseñanza/aprendizaje de lenguas, con temáticas en estrecha relación con el entorno inmediato de los aprendientes. En estos diálogos, claro que se pone el acento tanto en las estructuras gramaticales, como en los recursos educativos, literarios y culturales. Entonces, se nota que es imprescindible que la lengua y la literatura se aprendan simultáneamente, para que adquieran los alumnos las herramientas lingüísticas y culturales arriaba mencionadas. Otra vez, González Nieto (op. Cit.: 333) apunta en el mismo orden de ideas: 
Si se aceptan los supuestos de una perspectiva pragmática y discursiva, (...) la enseñanza de la literatura es ante todo, un aspecto parcial de la formación lingüística de los alumnos, sin perjuicio de que se pueden concretar, además, algunos fines específicos. Si la educación lingüística debe proponerse el desarrollo de unas capacidades en todos los ámbitos de la comunicación verbal, la literatura es un ámbito o un uso secundario de la lengua delimitado históricamente por una tradición (...). El valor educativo de los discursos reconocidos como literarios radica en que su potencial semántico, semiótico y expresivo, o retórico, por cuanto cumplen unas funciones comunicativas y cognitivas diferentes a las de la comunicación práctica.

En las líneas arriba mencionadas, se aclaran las relaciones entre la lengua y la literatura que se deberían enseñar a los alumnos. Ya hemos señalado que la lengua conlleva la cultura. Pues, la literatura es uno de los componentes esenciales de la cultura a través de la cual se identifican los ciudadanos de una región o de un país. A partir de este concepto, conviene volver a los fines y objetivos que se quieren alcanzar mediante la enseńanza-aprendizaje de una lengua-cultura extranjera, y poner de relieve la opinión de Girard (1972: 92) que estipula que: «l'enseignement de la langue n'est pas une fin en soi et que cet enseignement doit normalement déboucher sur la découverte d'une culture dont la langue constitue le support privilégié mais non unique». Con estas palabras, resumimos la definición de la lengua en dos aspectos:

\section{Lengua, instrumento de comunicación}

Se trata de la lengua en su uso como herramienta que sirve en la interacción social. De hecho la lengua favorece contactos entre los seres humanos y ayuda a establecer relaciones y comunicaciones. También se puede decir que es una forma de comportamiento social que permite a la gente dialogar, expresar sus ideas, sus opiniones, sus necesidades. López Valero y Encabo Fernández (2002) recuerdan

que en toda comunicación existen dos interlocutores que actúan según el turno de palabra bien como emisor bien como receptor. De igual modo, existe un mensaje que queda envuelto en un código lingüístico dependiente de la lengua y de la cultura en las que el proceso comunicativo se desarrolle. También habrá un canal (aire, medios de comunicación...) que se constituirá en el medio a través del cual el mensaje llegará a la persona receptora.

Señala el autor de esta cita que efectivamente un mensaje se vislumbra a través del código lingüístico —entre otros- Esto significa que aparte de la comunicación, la lengua desempeña otro papel como evocado arriba en la breve definición que hemos apuntado. Es el papel del vehículo de la cultura.

\section{Lengua, soporte cultural}

Cabe siempre poner de relieve las estrechas relaciones entre, la lengua y la cultura como conceptos indisociables. Cada vez que se intenta definir la cultura, cabe necesariamente insistir sobre el papel de la lengua a través de la cual el individuo expresa sus pensamientos y sus emociones.

En cuanto a la literatura, aparece como una forma de expresión, uno de los aspectos culturales que abarca casi todos los aspectos de la vida: la política (organización territorial y administrativa de 
España y de otros países del mundo, partidos políticos...), la sociedad (los servicios públicos como la sanidad, la enseñanza, los medios de comunicación), la religión, la economía, etc., de ahí, su carácter muy popular. Cuenta con dos ramas distintas: la literatura escrita y la literatura oral. Ahí, la lengua aparece como soporte cultural como lo presenta Girard (1972) en los aspectos oral y escrito:

Oral: Contenido lingüístico, mensajes orales con contenido cultural (científico, técnico, moral, religioso...), literatura únicamente oral (cuentos, leyendas, poemas, cantos, etc.), forma oral de la literatura escrita (poesía, teatro, etc.), obras radiofónicas o televisivas, cine.

Escrito: prensa, reportajes, estudios técnicos y científicos, estudios críticos, literatura escrita (todos los géneros).

Con todo lo anterior, vemos que la literatura tiene un papel de suma trascendencia en la enseñanza-aprendizaje de una lengua-cultura extranjera. Por consiguiente, conviene saber cómo integrarla en la clase de lengua, para un buen desarrollo de la formación lingüística y cultural de los alumnos de español lengua extranjera. El mismo Girard (1972) hizo un estudio minucioso de este proceso y propuso tres etapas para conseguirlo:

1. Dominar el lenguaje oral a través de los diálogos de la vida cotidiana.

2. Dominar el lenguaje escrito mediante la transcripción de los diálogos y relatos siempre privilegiando el componente oral

3. Iniciar a los alumnos a la literatura contemporánea intentando favorecer el pase de la lengua oral a la literatura contemporánea.

La propuesta de Girard se ve limitada en el tiempo. La ciencia evoluciona, y se sabe que hubo un cambio de enfoque metodológico en la enseñanza/aprendizaje de la lengua y la literatura en clases de lengua extranjera. Muchos estudios ya fueron realizados en este ámbito y concuerdan en que no se debería disociar la lengua y la literatura en la didáctica de la lengua. En esta reflexión, compartimos el punto de vista de Mendoza Fillola et al. (1996: 220). Piensan que "competencia lingüística y competencia literaria se dan relaciones de paralelismo, de dependencia (de la competencia literaria respecto a la lingüística), de intersección e, incluso, de lo que podría considerarse como desviación I disyunción". La competencia comunicativa integra varios aspectos tales como: el dominio de la competencia lingüística y de la competencia literaria. Por consiguiente, nos parece importante considerar otra definición de la literatura, la de Romera Castillo (1992: 138), en que especifica que:

Precisando más, podemos definirla como el conjunto de obras literarias. Una obra literaria es una ordenada serie de pensamientos, expresados por medio del lenguaje, y dirigida a un fin, que en último resultado nunca debe de ser otro que el bien de la especie humana. (...) En la anatomía especial de la obra literaria encontramos un cuerpo (unas ideas) recubierto por una serie de vestidos de más o menos calidad y variedad (recursos de lenguaje). De ahí que en la literatura podamos distinguir dos aspectos: los contenidos de ideas, los recursos de lenguaje.

También nos interesa hoy en día la opinión de otro investigador que reconoce que el objetivo primordial en la enseñanza de la literatura es el desarrollo de la competencia literaria que incluye otras herramientas importantes en la formación de los alumnos, como la lengua, la cultura y la historia. Desde esta reflexión, González Nieto (2001: 334-335), explica que: 
La enseñanza de la literatura debe concebirse, por lo tanto, como una ampliación de los saberes textuales, retóricos y expresivos y del sentido de la adecuación a la multiplicidad de situaciones e intenciones. Y también como una ampliación del mundo mental y cultural de los alumnos. Las formas literarias son inseparables de tales significados y ellas también significan, ya que la historia de la cultura y de la lengua es la de la evolución de unos significados y de unas formas de expresarlos.

Para conseguir esta meta, hay que tener en cuenta que los objetivos fijados dependen del nivel de los alumnos y de cada curso. En general, las actividades que se llevan a cabo deben favorecer la recepción, la comprensión, la lectura y el comentario para el desarrollo de las habilidades comunicativas. En todo caso, todos los actores presentes en una clase de lengua extranjera tienen que participar en su realización efectiva. Por eso, cabe recordar que "la enseñanza debe servir de guía, a los alumnos y orientarlos con un método coherente con nuestra propia concepción de la literatura”, según afirma González Nieto (2001).

La base teórica de algunas ciencias del lenguaje y sus relaciones con la didáctica que acabamos de presentar nos lleva a pensar en la manera cómo se diseñan e imparten las clases de español lengua extranjera en algunos contextos. De hecho, cabe señalar que la alusión al diseño de la clase de lengua española por el profesor remite a su propia formación. Al menos, suele ser una consecuencia de esta formación anteriormente recibida. No obstante, mencionamos de paso la ventaja que saca un profesor de lengua extranjera, al dominar el análisis del discurso, de la conversación, y de las distintas ciencias del lenguaje que le permiten enseñar su asignatura con maestría y, por ende, iniciar a sus alumnos en la comprensión y expresión de técnicas de interacción comunicativa.

Nos llama particularmente la atención este desarrollo sobre la didáctica de la lengua extranjera y la literatura. Lengua y literatura constituyen los dos principales pilares de la clase de lengua extranjera en la programación didáctica de toda lengua. Desde esta perspectiva, y teniendo en cuenta los actuales aspectos de la enseñanza-aprendizaje de una lengua extranjera, resaltamos la necesidad de garantizar a los alumnos la formación lingüística, sociocultural, discursiva, referencial y estratégica, evitando una enseñanza basada en la dicotomía Lengua/Literatura. Los dos conceptos se complementan, favorecen el desarrollo intelectual y la inserción social de los alumnos. Por otra parte, Lomas (1999) nos aconseja que no olvidemos que "el conocimiento de los textos literarios favorece un uso oral y escrito correcto ya que la literatura es el modelo de lengua que debe ser interiorizado e imitado por los alumnos (...) para hablar y escribir correcta y creativamente". Conforme se ha dicho en las tres etapas del proceso de enseñanza propuesto por Girard y, teniendo en cuenta las nuevas orientaciones de la didáctica de la lengua y la literatura, y la opinión de Lomas más arriba mencionada, la didáctica de las lenguas extranjeras en Camerún se transformaría considerablemente, mejorando así la labor educativa a la que nos dedicamos y, sobre todo la formación lingüística y cultural de los aprendientes.

En este proceso, no sería inútil recordar el fuerte e imborrable papel de la metodología. La enseñanza-aprendizaje de una lengua extranjera implica tanto al profesor, como a los alumnos; y condiciona los materiales que sirven a la puesta en práctica de los contenidos lingüísticos, y al contexto en que se desarrolla dicho proceso. Con esta situación, volvemos al importante problema de la teoría y la práctica, en la medida en que el profesor dispone de la teoría por una parte, y por otra, su encuentro con los alumnos, en el aula, le permite poner en aplicación su diseño, con el apoyo de los materiales didácticos. Se trata de lo que Chevallard (1991) llama la transposición didáctica (TD). En resumen, la transposición didáctica se define como el paso del saber teórico al saber enseñado. En otras palabras, es el conjunto de procesos de transformaciones del saber 
científico teórico en avatares didácticos y permite la articulación del análisis epistemológico con el análisis didáctico. A partir de esta consideración y dada la variedad de los contextos en que tiene lugar la enseñanza de una lengua extranjera, hay que pensar en el procedimiento que se debe adoptar durante la práctica docente. En este sentido, cabe recordar aquí la necesidad de tener en cuenta el diseño y la manera concreta de proceder en el aula, para impartir una clase de lengua extranjera. Sánchez Pérez (1997) hace hincapié en la misma preocupación cuando nos aconseja:

El mejor bagaje de un profesor que inicia su camino o quiere consolidar su futuro profesional es una sólida formación sobre todos los componentes que participan en la docencia. Desde esta perspectiva es inútil y sobre todo estéril cualquier minusvaloración de la teoría en contraposición a la práctica. La teoría y la práctica son dos caras de la misma moneda. Como afirma Wardhaugh (1969): No hay nada tan práctico como una buena teoría.

La teoría y la práctica docente se refieren al profesor y a los alumnos como agentes que participan en el desarrollo del proceso educativo. No obstante, existen otros aspectos que contribuyen a la efectividad del mismo. Uno de ellos se denomina la metodología, y es fuente de diversas interpretaciones. La metodología forma parte de los elementos fundamentales del proceso didáctico. Se refiere al procedimiento, a los materiales didácticos utilizados, al cómo hacer en el aula. En el marco de la enseńanza-aprendizaje de una lengua extranjera, nos referimos a la siguiente consideración de Sánchez Pérez (1993) que nos parece más apropiada:

Utilizar una metodología en una clase de idiomas equivale a elegir un camino determinado para la docencia, frente a otros posibles a que el profesor tiene opción. Pues bien, la elección consciente y razonada, así como la configuración de un camino o método a través del cual se pretende la consecución de objetos concretos se lleva a cabo tomando en cuenta tres ejes: La naturaleza y característica de la lengua objeto de la enseńanza, junto con las creencias y convicciones respecto a ella. Las características de los objetivos que pretende alcanzarse (concretados en el contenido que debe aprenderse). Los instrumentos, medios o técnicas de que nos valdremos para alcanzar tales objetivos.

Como notamos en esta cita, el uso de una metodología en la enseñanza-aprendizaje de una lengua extranjera designa la complejidad de la actuación docente, y se presenta como un todo coherente, un conjunto armonizado en el que vienen implicados estos elementos enumerados al final de la recién citada opinión de Sánchez Pérez. El deseo de aprender las lenguas-culturas extranjeras o en una palabra, ser multilingüe es una obligación, si se quiere garantizar la inserción social. De ahí, el afán que experimentan los profesionales docentes de encontrar el camino, el método más seguro para el aprendizaje de dichos idiomas. De hecho, una de las principales preocupaciones de los docentes que imparten clases se resume en la pregunta ¿Cómo hacer en el aula? Para los alumnos, el cómo precisa los caminos adecuados que hay que recorrer para asegurar el aprendizaje. También se suelen preguntar los docentes qué hace falta hacer, para referirse a los contenidos específicos que deben elegir. En los dos casos, se trata del método que según Nussbaum y Bernaus (2001) "se define como una aplicación de técnicas concretas de enseñanza, basadas en ciertos principios teóricos que se plasman en un programa único". Los métodos de enseńanza de la lengua dentro del aula deberían también tener como base la lingüística funcional como sugiere Manga (2014:20), ya que ésta insiste sobre la necesidad de analizar la lengua desde la conceptualización hasta su entorno de producción y uso, con la integración esencial de los aspectos etnológicos y culturales, a través de los cuales 
la lengua cumple sus funciones primordiales, y asegura la comunicación social. En cada proceso didáctico, siempre es indispensable respetar el programa previsto, es decir, una planificación de contenidos precisos y actividades de clase, con la pretensión de alcanzar unos objetivos específicos susceptibles de contribuir en la formación de los alumnos que nos confía la sociedad. En este sentido, Candlin (1981:9) distingue cinco componentes de la programación didáctica que cabe tomar en consideración:

a. Finalidad general, p. ej., desarrollo de la personalidad del alumno como individuo y como ser social, potenciando su competencia comunicativa, su capacidad crítica, su empatía con la comunidad de la LM...

b. Planificación del proceso instructivo: metas didácticas y objetivos educativos. Análisis de los intereses y necesidades del GM (...)

c. Enseñanza. Decisiones de tipo pedagógico y metodológico; rasgos del carácter del profesor y del alumno; hábitos de enseñanza y de aprendizaje; factores espaciotemporales, objetivos de la enseñanza; motivación...

d. Diseño de tareas. Complejidad de los aspectos formal y funcional de la lengua, técnicas de estudio, competencia comunicativa, las cuatro destrezas (comprensión auditiva y lectora, expresión oral y escrita), argumentación...

e. Evaluación. Exámenes, ejercicios en clase, evaluación personal, retroalimentación (feedback) tras la realización de las tareas, fichas de trabajo...

En otras palabras, a través de todas las actividades de clase, el docente debe intentar respetar la interdependencia entre la lengua y la literatura para entrenar a sus alumnos en la producción oral y escrita en la lengua meta, y en la apropiación de la competencia comunicativa y cultural.

\section{Conclusión}

Se suele repetir que los que se desenvuelven en el aprendizaje de una lengua extranjera tienen como razón fundamental el deseo de expresarse en dicha lengua. Aprender lengua y literatura extranjeras desempeña importantes roles en la formación moral e intelectual del alumno, y le permite consolidar su identidad sociocultural facilitándole del mismo modo contactos con otros pueblos en este mundo globalizado. Pero cabe subrayar que todo depende del contexto en que se realizan los estudios o la adquisición del idioma. Nosotros nos dedicamos a la enseñanza/aprendizaje de las lenguas en general y, el español como lengua extranjera nos llama particularmente la atención. Ya sugerimos a los futuros docentes que el registro formal debe ser prioritario en el desarrollo de sus actividades de clases. Pues, es necesario actualizar frecuentemente sus conocimientos, adaptarse a los cambios metodológicos adecuados, y confirmar que el dominar únicamente la sub-competencia lingüística no basta en el aprendizaje de una lengua extranjera. Existen otras sub-competencias (sociolingüística, discursiva, estratégica, sociocultural, social). El currículum oficial para la enseńanza/aprendizaje de las lenguas en Camerún recomienda a los docentes iniciar a los alumnos en lengua, literatura y civilización como aspectos esenciales en la formación de los individuos. Las enseñanzas de la lengua y la literatura enriquecen las capacidades comunicativas y socio-culturales de los aprendientes, y les ayudan a comprender fácilmente los mensajes de sus interlocutores, y a expresarse en la lengua meta. De esta manera, se adquieren habilidades comprensivas y expresivas; así como otras destrezas que se necesitan para adaptarse en distintos contextos de la comunicación social. 


\section{Referencias bibliográficas}

CANDLIN N. C.., The communicative teaching of English. Principles and exercise typology, Longman, London, 1981.

CHEVALLARD Y., La transposición didáctica, Del saber sabio al saber enseñado, Aique, Buenos Aires, 1997.

CORTÉS MORENO M., Guía para el profesor de idiomas, Didáctica del español y segundas lenguas, Octaedro, Barcelona, 2000.

GONZÁLEZ NIETO, L., Teoría lingüistica y enseñanza de la lengua. Lingüistica para profesores, Cátedra, Madrid, 2001.

GIRARD D., Linguistique appliquée et Didactique des langues, Armand Colin, Paris, 1972.

LÁZARO CARRETER, F. y CORREA CALDERÓN E., Cómo se comenta un texto literario, Cátedra, Madrid, 1996.

LOI no 98/004 DU 14 avril 1998 D'Orientation de l'Education au Cameroun. LOMAS, C., Cómo enseñar a hacer cosas con las palabras. Teoría y práctica de la educación lingüistica, Vol. 1, Paidós, Barcelona, , 1999.

LOMAS, C., Cómo enseñar a hacer cosas con las palabras. Teoría y práctica de la educación lingüistica, Vol. 2, Paidós, Barcelona, 1999.

LÓPEZ VALERO, A. y ENCABO FERNÁNDEZ, E. Introducción a la didáctica de la lengua y la literatura, un enfoque sociocrítico, Octaedro, Barcelona, 2002.

MANGA, A.M., Didáctica de lenguas extranjeras. Orientaciones teóricas en español, L'Harmattan, Paris, (2014)

MENDOZA FILLOLA, A. et al., Didáctica de la lengua para la enseñanza primaria y secundaria, Akal, Madrid, 1996.

NUSSBAUM, L. y BERNAUS, M., Didáctica de las lenguas extranjeras en la Educación Secundaria obligatoria, Síntesis educación, Madrid, 2001.

Programa de enseñanzalaprendizaje del español como lengua extranjera en la Enseñanza Secundaria en Camerún, (2000).

Programmes. Langue et Littérature 2nd Cycle (1994).

ROMERA CASTILLO, J., Didáctica de la lengua y la literatura. Método y práctica, Editorial Playor, Madrid, 1992.

SÁNCHEZ PÉREZ, A., Hacia un método integral en la enseñanza de idiomas. Estudio analítico, SGEL, Madrid, 1993.

SÁNCHEZ PÉREZ, A., Los métodos en la enseñanza de idiomas. Evolución histórica y análisis didáctico, SGEL, Madrid, 1997. 